\title{
Strong earthquake activity all over the world and strong-moderate earthquake ac- tivity within and near China (December 2007 January 2008)
}

\author{
CHEN Pei-shan (陈培善) \\ Institute of Geophysics, China Earthquake Administration, Beijing 100081, China \\ CLC number: P315.5 Document code: $\mathrm{R}$
}

\section{Illustration}

All the data in this catalog are chosen from the "Preliminary Seismological Report of Chinese Seismic Stations" (Its abbreviation is "Monthly Report"). The catalog includes the events of $M \geq 4.7$ in and near China and $M \geq 6$ all over the world. The "Monthly Report" is monthly compiled by the Ninth Section of Institute of Geophysics, China Earthquake Administration.

The origin times of earthquakes in the catalog adopt coordinated universal time (UTC) in accordance with international convention. The location of every epicenter is expressed by its latitude and longitude, at the same time by the corresponding geographical region proposed by Flinn and Engdahl. The regional names are only for references and do not imply any political significance. The number of stations used and standard deviation are also given in order to illustrate the precision of location.

The surface wave magnitude $M_{\mathrm{S}}$ is measured from the records of intermediate period broad band SK seismographs and adopting the surface wave magnitude formula of Beijing Station of 1965: $M_{\mathrm{S}}=\lg \left(A_{\mathrm{H}} / T\right)+1.66 \lg \Delta+3.5\left(1^{\circ}<\Delta<130^{\circ}\right)$, in which $A_{\mathrm{H}}$ is the resultant displacement amplitude of the maximum surface wave of horizontal components. $M_{\mathrm{S} 7}$ is measured from records of the long-period seismographs of 763 type and adopting the surface wave magnitude formula recommended by IASPEI in 1967. $M_{\mathrm{S} 7}=\lg \left(A_{\mathrm{V}} / T\right)+1.66 \lg \Delta+3.3\left(20^{\circ}<\Delta<160^{\circ}\right)$, in which $A_{\mathrm{V}}$ is the maximum ground displacement of surface wave in vertical component, $m_{\mathrm{b}}$ is short-period body-wave magnitude. $M_{\mathrm{L}}$ is local magnitude. In order to avoid confusion, no conversion is made among the various magnitudes. For convenience of use and comparison, the surface wave magnitude $M_{\mathrm{SZ}}$ (NEIS) and $m_{\mathrm{b}}$ (NEIS) measured by NEIS recorded on short period seismographs are also listed. 
Catalog of earthquakes within and near China

(December 2007 January 2008; $M \geq 4.7$ )

\begin{tabular}{|c|c|c|c|c|c|c|c|c|c|c|c|c|c|c|}
\hline \multirow{3}{*}{ No. } & \multirow{2}{*}{\multicolumn{2}{|c|}{$\begin{array}{l}\text { Origin time } \\
\text { (UTC) }\end{array}$}} & \multirow{2}{*}{\multicolumn{2}{|c|}{$\begin{array}{l}\text { Geographic } \\
\text { coordinates }\end{array}$}} & \multirow{3}{*}{$\begin{array}{c}\text { Focal } \\
\text { depth } \\
/ \mathrm{km}\end{array}$} & \multicolumn{6}{|c|}{ Magnitudes } & \multirow{3}{*}{ SD } & \multirow{3}{*}{$\begin{array}{c}\text { No. } \\
\text { sta. } \\
\text { used }\end{array}$} & \multirow{3}{*}{ Region } \\
\hline & & & & & & & & nina & & $\mathrm{NE}$ & & & & \\
\hline & $\mathrm{d}$ & h:min:s & $\varphi_{\mathrm{N}} /{ }^{\circ}$ & $\lambda_{\mathrm{E}} /{ }^{\circ}$ & & $M_{\mathrm{S}}$ & $M_{\mathrm{S} 7}$ & $M_{\mathrm{L}}$ & $m_{\mathrm{b}}$ & $M_{\mathrm{SZ}}$ & $m_{\mathrm{b}}$ & & & \\
\hline \multicolumn{15}{|c|}{ December 2007} \\
\hline 1 & 4 & $12: 31: 18.0$ & 22.05 & 111.49 & 22 & 4.1 & 4.0 & 4.6 & 4.8 & & & 2.5 & 16 & Eastern China \\
\hline 2 & 5 & $01: 41: 42.5$ & 23.24 & 121.15 & 10 & 4.8 & 4.7 & 4.8 & 4.8 & & 5.0 & 1.6 & 72 & Taiwan \\
\hline 3 & 5 & $04: 33: 37.2$ & 25.76 & 124.33 & 204 & & & & 5.2 & & 5.1 & 1.1 & 77 & North-east of Taiwan \\
\hline 4 & 8 & $16: 51: 58.2$ & 24.00 & 121.90 & 10 & & & 3.6 & 4.7 & & 4.5 & 1.6 & 6 & Taiwan \\
\hline 5 & 11 & $15: 56: 37.7$ & 35.12 & 77.41 & 19 & 4.7 & 4.5 & 4.9 & 4.8 & & 5.2 & 1.3 & 66 & Eastern Kashmir \\
\hline 6 & 16 & $08: 23: 36.2$ & 41.44 & 131.42 & 576 & & & & 5.0 & & 4.5 & 0.9 & 22 & Sea of Japan \\
\hline 7 & 17 & $07: 30: 47.5$ & 39.81 & 75.08 & 50 & 4.3 & 3.9 & 4.4 & 4.7 & & 4.6 & 1.7 & 41 & Southern Xinjiang \\
\hline 8 & 30 & $09: 55: 32.7$ & 36.50 & 84.59 & 42 & 4.9 & 4.5 & 5.0 & 4.6 & & 4.5 & 1.8 & 52 & Southern Xinjiang \\
\hline \multicolumn{15}{|c|}{ January 2008} \\
\hline 9 & 9 & $08: 26: 44.0$ & 32.39 & 85.27 & 10 & 6.8 & 6.6 & & 5.9 & 6.4 & 6.3 & 1.3 & 84 & Tibet \\
\hline 10 & 9 & $09: 32: 40.3$ & 32.23 & 84.90 & 8 & 4.7 & 4.6 & & 4.3 & & 4.2 & 2.1 & 28 & Tibet \\
\hline 11 & 14 & $03: 21: 43.2$ & 32.54 & 85.26 & 22 & 4.7 & 4.5 & & 4.5 & & 4.9 & 1.6 & 50 & Tibet \\
\hline 12 & 16 & $11: 54: 43.4$ & 32.44 & 85.18 & 10 & 6.2 & 6.1 & & 5.4 & 5.8 & 6.0 & 1.5 & 80 & Tibet \\
\hline 13 & 17 & $06: 25: 42.9$ & 43.62 & 84.31 & 13 & 4.4 & 4.1 & 4.9 & 4.5 & 4.1 & 4.8 & 1.9 & 54 & Northern Xinjiang \\
\hline 14 & 17 & 07:09:56.1 & 32.34 & 85.15 & 16 & 4.7 & 4.5 & & 4.6 & & 4.9 & 1.9 & 48 & Tibet \\
\hline 15 & 18 & $19: 18: 50.1$ & 38.18 & 73.22 & 47 & 4.4 & 4.1 & 4.7 & 4.8 & & 4.7 & 1.3 & 45 & Tadzhikistan \\
\hline 16 & 22 & $18: 43: 32.4$ & 32.49 & 85.29 & 13 & 5.6 & 5.4 & & 5.1 & 5.2 & 5.6 & 1.2 & 80 & Tibet \\
\hline 17 & 31 & $21: 06: 47.1$ & 28.41 & 105.01 & 30 & 4.6 & 4.3 & 4.7 & 4.5 & & 4.8 & 1.9 & 71 & Yunnan \\
\hline
\end{tabular}

Catalog of earthquakes all over the world

(December 2007 January 2008; $M \geq 6.0$ )

\begin{tabular}{|c|c|c|c|c|c|c|c|c|c|c|c|c|c|}
\hline \multirow{3}{*}{ No. } & \multirow{2}{*}{\multicolumn{2}{|c|}{$\begin{array}{l}\text { Origin time } \\
\text { (UTC) }\end{array}$}} & \multirow{2}{*}{\multicolumn{2}{|c|}{$\begin{array}{l}\text { Geographic } \\
\text { coordinates }\end{array}$}} & \multirow{3}{*}{$\begin{array}{c}\text { Focal } \\
\text { depth } \\
/ \mathrm{km}\end{array}$} & \multicolumn{5}{|c|}{ Magnitudes } & \multirow{3}{*}{$\mathrm{SD}$} & \multirow{3}{*}{$\begin{array}{c}\text { No. } \\
\text { sta. } \\
\text { used }\end{array}$} & \multirow{3}{*}{ Region } \\
\hline & & & & & & \multicolumn{3}{|c|}{ China } & \multicolumn{2}{|c|}{ NEIS } & & & \\
\hline & $\mathrm{d}$ & h:min:s & $\varphi /^{\circ}$ & $\lambda /{ }^{\circ}$ & & $M_{\mathrm{S}}$ & $M_{\mathrm{S} 7}$ & $m_{\mathrm{b}}$ & $M_{\mathrm{SZ}}$ & $m_{\mathrm{b}}$ & & & \\
\hline \multicolumn{14}{|c|}{ December 2007} \\
\hline 1 & 9 & $07: 28: 20.8$ & $26.00 \mathrm{~S}$ & $177.50 \mathrm{~W}$ & 152 & & & 6.6 & & 7.0 & 2.2 & 74 & South of Fiji \\
\hline 2 & 13 & $07: 23: 45.6$ & $23.14 \mathrm{~S}$ & $70.74 \mathrm{~W}$ & 58 & 6.2 & 6.2 & & 5.9 & 5.4 & 2.4 & 62 & Off coast of Northern Chile \\
\hline 3 & 13 & $15: 51: 27.0$ & $15.19 \mathrm{~S}$ & $172.42 \mathrm{~W}$ & 17 & 5.7 & 5.5 & 5.7 & 6.0 & 6.0 & 1.0 & 77 & Samoa region \\
\hline 4 & 16 & 08:09:17.9 & $23.00 \mathrm{~S}$ & $70.20 \mathrm{~W}$ & 45 & 6.8 & 6.8 & & 6.4 & 6.2 & 1.9 & 75 & Near coast of Northern Chile \\
\hline 5 & 19 & 09:30:24.6 & $51.34 \mathrm{~N}$ & $179.52 \mathrm{~W}$ & 29 & 6.8 & 6.7 & 6.2 & 7.1 & 6.4 & 1.0 & 85 & Andreanof Islands \\
\hline 6 & 19 & $09: 44: 19.0$ & $51.10 \mathrm{~N}$ & $179.30 \mathrm{~W}$ & 47 & 6.0 & 5.5 & 5.4 & & 5.4 & 1.3 & 52 & Andreanof Islands \\
\hline 7 & 20 & $07: 55: 13.3$ & $38.74 \mathrm{~S}$ & $178.64 \mathrm{E}$ & 22 & 6.6 & 6.4 & 5.8 & 6.5 & 6.3 & 1.3 & 66 & Off east coast of North Islands, N.Z. \\
\hline 8 & 21 & $07: 23: 33.6$ & $51.53 \mathrm{~N}$ & $179.11 \mathrm{~W}$ & 25 & 6.0 & 5.8 & 5.6 & & 5.7 & 1.1 & 73 & Andreanof Islands \\
\hline 9 & 21 & $07: 24: 32.5$ & $51.17 \mathrm{~N}$ & $179.22 \mathrm{~W}$ & 26 & 6.1 & 5.9 & 6.1 & & 6.1 & 1.3 & 69 & Andreanof Islands \\
\hline 10 & 22 & 07:11:07.3 & $2.48 \mathrm{~S}$ & $139.29 \mathrm{E}$ & 37 & 6.0 & 5.9 & 5.6 & 6.1 & 6.0 & 0.9 & 85 & West Irian \\
\hline 11 & 22 & $12: 26: 15.1$ & $1.90 \mathrm{~N}$ & $96.82 \mathrm{E}$ & 33 & 6.2 & 6.0 & 5.4 & 5.9 & 5.6 & 1.0 & 84 & Off west coast of Northern Sumatera \\
\hline 12 & 26 & $22: 04: 55.6$ & $52.82 \mathrm{~N}$ & $168.79 \mathrm{~W}$ & 34 & 6.5 & 6.4 & 5.8 & 6.2 & 6.0 & 0.9 & 84 & Fox Islands \\
\hline 13 & 29 & $22: 58: 05.4$ & $52.70 \mathrm{~N}$ & $168.78 \mathrm{~W}$ & 36 & 6.0 & 5.8 & 5.6 & 5.6 & 5.7 & 1.0 & 84 & Fox Islands \\
\hline \multicolumn{14}{|c|}{ January 2008} \\
\hline 14 & 1 & $18: 54: 56.0$ & $6.31 \mathrm{~S}$ & $147.39 \mathrm{E}$ & 54 & 5.8 & 5.7 & 6.0 & 6.1 & 6.0 & 0.8 & 85 & Eastern New Guinea region \\
\hline 15 & 4 & $07: 29: 14.6$ & $3.11 \mathrm{~S}$ & $100.89 \mathrm{E}$ & 41 & 6.0 & 5.9 & 5.7 & 5.9 & 5.9 & 1.1 & 81 & Southern Sumatera \\
\hline 16 & 5 & $11: 01: 02.3$ & $51.30 \mathrm{~N}$ & $130.80 \mathrm{~W}$ & 10 & 7.0 & 6.7 & 5.5 & 6.5 & 6.0 & 1.5 & 78 & Vancouver Islands region \\
\hline 17 & 5 & $11: 44: 44.6$ & $51.20 \mathrm{~N}$ & $130.50 \mathrm{~W}$ & 10 & 6.7 & 6.4 & 5.6 & 6.5 & 6.0 & 1.6 & 68 & Vancouver Islands region \\
\hline 18 & 6 & $05: 14: 11.5$ & $37.16 \mathrm{~N}$ & $22.25 \mathrm{E}$ & 54 & 5.7 & 5.5 & 6.2 & & 6.1 & 0.6 & 80 & Southern Greece \\
\hline 19 & 9 & $08: 26: 44.0$ & $32.39 \mathrm{~N}$ & $85.27 \mathrm{E}$ & 10 & 6.8 & 6.6 & 5.9 & 6.4 & 6.3 & 1.3 & 84 & Tibet \\
\hline 20 & 9 & $14: 40: 01.2$ & $52.06 \mathrm{~N}$ & $132.13 \mathrm{~W}$ & 10 & 6.2 & 6.0 & 5.0 & 5.9 & 5.5 & 1.3 & 74 & Queen Charlotte Islands region \\
\hline 21 & 10 & $01: 37: 16.5$ & $43.80 \mathrm{~N}$ & $127.30 \mathrm{~W}$ & 10 & 6.4 & 6.0 & 5.3 & 5.9 & 5.6 & 1.8 & 77 & Off coast of Oregon \\
\hline 22 & 16 & $11: 54: 43.4$ & $32.44 \mathrm{~N}$ & $85.18 \mathrm{E}$ & 10 & 6.2 & 6.1 & 5.4 & 5.8 & 6.0 & 1.5 & 80 & Tibet \\
\hline 23 & 20 & $20: 26: 02.2$ & $2.18 \mathrm{~N}$ & $126.82 \mathrm{E}$ & 43 & 5.6 & 5.5 & 6.0 & 5.5 & 6.0 & 1.0 & 85 & Molucca Passage \\
\hline 24 & 22 & $07: 55: 48.8$ & $15.30 \mathrm{~S}$ & $175.30 \mathrm{~W}$ & 6 & 6.1 & 5.8 & 5.1 & 6.0 & 5.6 & 2.7 & 59 & Tonga \\
\hline 25 & 22 & $10: 49: 21.6$ & $14.41 \mathrm{~S}$ & $174.65 \mathrm{~W}$ & 10 & 6.1 & 5.9 & 5.1 & 6.0 & 5.7 & 2.0 & 61 & Samoa region \\
\hline 26 & 22 & $17: 14: 47.7$ & $0.66 \mathrm{~N}$ & $97.40 \mathrm{E}$ & 10 & 6.5 & 6.4 & 5.6 & & 5.4 & 0.9 & 66 & Off west coast of Northern Sumatera \\
\hline 27 & 22 & $17: 14: 56.9$ & $1.00 \mathrm{~N}$ & $97.40 \mathrm{E}$ & 20 & 6.6 & 6.5 & 5.7 & & 5.9 & 2.0 & 33 & Off west coast of Northern Sumatera \\
\hline
\end{tabular}

